\title{
Flat grinding model, considering internal dynamics of the process
}

\author{
Sergey Bratan ${ }^{1, *}$, and Ekaterina Vladetskaya ${ }^{1}$ \\ ${ }^{1}$ Sevastopol State University, Department of Mechanical Engineering, 299053 Sevastopol, Russia
}

\begin{abstract}
The article considers the results of theoretical studies of the process of flat external grinding on the basis of the developed dynamic model. In order to take into account the influence of the statistical properties of the current deviations of the radius vectors of the grinding wheel and the thickness of the work piece, in addition to the input signal, a random process with the same stochastic characteristics as that of the current deviation of the radius vector of the grinding wheel and the billet topography is fed to the input of the system model. The corresponding random input signal can be obtained by means of a shaping filter. The output coordinate of the shaping filter according to its statistical parameters corresponds to the parameters of the components of the dynamic system characterizing the processes of the grinding wheel effect on the work piece during the grinding process. The developed mathematical model of the operation of flat external grinding can be used to determine the estimates of the quality characteristics of machined parts and systems for automatic control of the process of flat external grinding when the model is supplemented by dynamic links characterizing the statistical properties of the grinding wheel and work piece.
\end{abstract}

\section{Introduction}

The quality of the processed products depends to a large extent on the quality of the workpiece, the processing technology, the dynamics of the machine, and the quality of the tools. Studies of grinding process show that, as a rule, at the initial moment of time, with the correct selection of tool characteristics, cutting conditions, and the optimal construction of the grinding cycle, the specified parameters of accuracy and surface roughness of the workpiece are provided $[1,2]$.

Of all the elements of the technological system, only the grinding wheel and the work piece undergo substantial changes in a relatively short time interval [3]. The change in the state of the tool has a significant effect on the course of the technological process, since the tool profile is copied on the surface of the workpiece. For example, an increase in the deviation of the shape of a circle is equivalent to a dynamic change in dimensional parameters, which leads to additional perturbations of the dynamic system of the machine, and, consequently, to an increase in the roughness and waviness of the part and the

* Corresponding author: serg.bratan@gmail.com 
formation of a defective layer on its surface [4-6]. Such changes in dimensional parameters at the end of the period of tool life may increase several times [7-10].

The presence of oscillations in deviations in the location and roughness of the surface is explained by the disturbing effects in technological processes caused by instability in the technological system. That is, the instability of the parameters of parts is determined by the impact in the process of their production on the technological system (TS) of changing external factors, some of which are unknown and not controlled during processing. This problem is especially important for finishing operations, on which the quality parameters of the finished products are finally formed and which are most sensitive to disturbing effects [11].

In modern conditions, about $15 \ldots 20 \%$ of finishing operations are performed by methods of flat external grinding. This determines the urgency of further improvement of such operations. The task of increasing their productivity while ensuring the stability of the required quality parameters, the geometry of the surface of the parts requires consideration of the influence of disturbing factors in the course of the finishing technological process of the operation of flat external grinding [12].

\section{Main text}

At present, flat external grinding is carried out using traditional methods that do not fully take into account the influence of random factors that reduce the stability of the quality indicators of manufactured products [13]. To stabilize the quality indicators, technological regimes are assigned, proceeding from unfavorable conditions, for example, the renewal of the cutting properties of a worn out grinding wheel is performed much earlier than required by its actual state. When predicting the state of the TS, traditional deterministic models of the technological process (TP) are used, using traditional cutting modes, methods of diagnostics and control [4].

At the same time, grinding processes have a complex stochastic nature, which leads to a dispersion of product quality indicators and does not allow using all the possibilities of finishing methods. Ensuring the quality and efficiency of the flat grinding operation is possible only on the basis of the development of dynamic models, taking into account and determining the parameters of the ongoing stochastic processes, which is the goal of this paper [5].

Accounting for changes and assessing the impact of disturbing factors on the technological process requires the construction of a mathematical description. This description should include dynamic models of the technological process and the corresponding mathematical models of the disturbing factors.

The dynamics of the process of flat external grinding is most fully reflected by the normal grinding force. The composition of such a force includes its average value and the components caused by the residual unbalance of the grinding wheel, as well as shape deviations of the tool and workpiece in the zone of their interaction.

The purpose of this article is to develop a mathematical model of a flat grinding operation that takes into account the internal dynamics of the process.

Fig. 1 shows an equivalent scheme of the dynamic system of a flat grinding machine.

The value of the average normal grinding force depends on the average values of the relationship between the center of the grinding wheel and the surface of the workpiece and is determined by technological regimes and parameters of rigidity $c_{1}$ and $c_{3}$ processing. In turn, it determines the average value of the movements of the elastic system of the machine, which can be determined and taken into account at the stage of development of the technological process. 


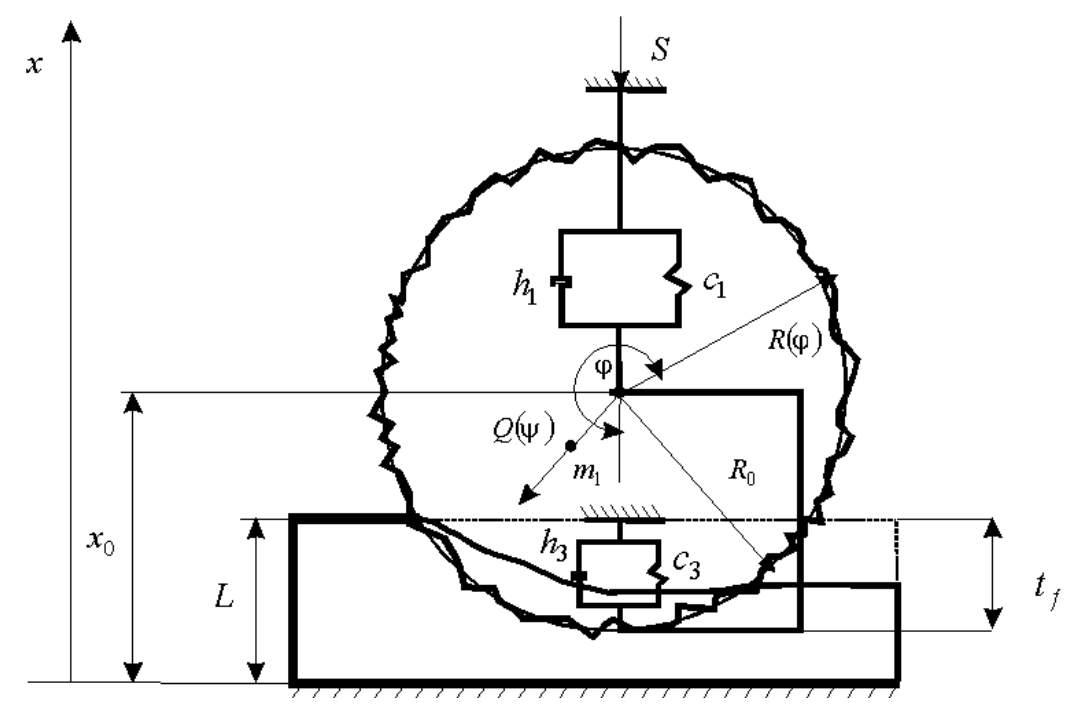

Fig. 1. Equivalent scheme of surface grinding machine dynamic system.

The components caused by the dynamic imbalance effects and shape deviations of the grinding wheel and workpiece lead to corresponding deviations from the nominal (projected) technological treatment regime and, consequently, to deviations in the quality of the treated surface.

With the use of the d'Alembert principle, taking into account the fact that the workpiece is rigidly fixed, the model of dynamics of the process of flat external grinding is presented in the form:

$$
m_{1} \ddot{x}+h_{1} \dot{x}+c_{1} x=h_{3}(\dot{L}+\dot{R}-\dot{x})+c_{3}(L+R-x)-c_{1} s-h_{1} \dot{s},
$$

where (in accordance with Fig. 1) $m_{1}$ - is the reduced mass of the grinding wheel and the grinding head; $h_{1}$ - reduced coefficient of damping of the grinding wheel; $c_{1}$ - reduced rigidity of the grinding wheel and grinding head; $h_{3}$ - damping of the contact area of the grinding wheel with the workpiece; $c_{3}$ - the stiffness of the contact zone of the grinding wheel with the workpiece; $L$ - thickness of the workpiece (distance from the table to the surface of the workpiece); $R$ - radius of the grinding wheel towards the workpiece; $x$ - is the distance from the basic surface to the center of rotation of the circle; $S$ - distance due to movement.

If there is no imbalance, when the center of rotation coincides with the center of mass, and the thickness of the part $L$ and the radius of the grinding wheel $R$ have deviations from the nominal values, i.e. $S=S_{0}+\Delta S, L=L_{0}+\Delta L, R=R_{0}+\Delta R$, the position of the grinding wheel also differs from the nominal value $x_{0}$, i.e., $x=x_{0}+\Delta x$ and relation (1) takes the form:

$$
\begin{aligned}
& m_{1}\left(\ddot{x}_{0}+\Delta \ddot{x}\right)+h_{1}\left(\dot{x}_{0}+\Delta \dot{x}\right)+c_{1}\left(x_{0}+\Delta x\right)=h_{3}\left(\dot{L}_{0}+\Delta \dot{L}+\dot{R}_{0}+\Delta \dot{R}-\dot{x}_{0}-\Delta \dot{x}\right)+ \\
& +c_{3}\left(L_{0}+\Delta L+R_{0}+\Delta R-x_{0}-\Delta x\right)-c_{1}\left(s_{0}+\Delta s\right)-h_{1}\left(\dot{s}_{0}+\Delta \dot{s}\right)
\end{aligned},
$$

Taking into account the substitution $\Delta x=0, \Delta L=0, \Delta R=0, \Delta s=0$ the following expression is obtained in relation (2):

$$
m_{1} \ddot{x}_{0}+h_{1} \dot{x}_{0}+c_{1} x_{0}=h_{3}\left(\dot{L}_{0}+\dot{R}_{0}-\dot{x}_{0}\right)+c_{3}\left(L_{0}+R_{0}-x_{0}\right)-c_{1} s_{0}-h_{1} \dot{s}_{0}
$$


Writing the difference between expressions (2) and (3), we construct the equation of motion of the center of the grinding wheel in the deviations:

$$
m_{1} \Delta \ddot{x}+h_{1} \Delta \dot{x}+c_{1} \Delta x=h_{3}(\Delta \dot{L}+\Delta \dot{R}-\Delta \dot{x})+c_{3}(\Delta L+\Delta R-\Delta x)-c_{1} \Delta s-h_{1} \Delta \dot{s}
$$

If the rotation of the grinding wheel is not carried out around the center of mass, then a force appears that characterizes the effect of the imbalance.

Taking into account the imbalance $Q(t)$, equality (4) takes the form:

$$
\begin{aligned}
& m_{1} \Delta \ddot{x}+h_{1} \Delta \dot{x}+c_{1} \Delta x=h_{3}(\Delta \dot{L}+\Delta \dot{R}-\Delta \dot{x})+c_{3}(\Delta L+\Delta R-\Delta x)- \\
& -c_{1} \Delta s-h_{1} \Delta \dot{s}+Q(t) .
\end{aligned}
$$

Rewriting expression (5) in Laplace images, we obtain:

$$
\begin{aligned}
& m_{1} p^{2} \Delta x+h_{1} p \Delta x+c_{1} \Delta x=h_{3}(p \Delta L+p \Delta R-p \Delta x)+ \\
& +c_{3}(\Delta L+\Delta R-\Delta x)-c_{1} \Delta s-h_{1} p \Delta s+Q(p),
\end{aligned}
$$

$Q(p)$ - the corresponding form of expression (6).

The force caused by the imbalance is:

$$
Q=P_{\max } \cdot \sin \left(\omega t+\varphi_{0}\right)=\frac{P_{\max } \cdot T_{2}}{1+T_{2}^{2} p^{2}} \cdot\left[T_{2} p \sin \varphi_{0}+\cos \varphi_{0}\right],
$$

where $T_{2}=1 / \omega ; \omega$ - rotational speed of the grinding wheel; $\varphi_{0}-$ the angle of the initial unbalance position relative to the point of contact with the workpiece.

It can be noted that the second factor of expression (7) in the simulation is responsible for the phase shift of the harmonic oscillations characterizing the effect of the imbalance effect of the grinding wheel corresponding to its initial position relative to the contact point.

The equation in the deviations in the operator form is written in the following form:

$$
d x=\frac{h_{3} p d L+h_{3} p d R+c_{3} d L+c_{3} d R-c_{1} d s-h_{1} p d s+Q}{m_{1} p^{2}+h_{1} p+h_{3} p+c_{1}+c_{3}} .
$$

To analyze the influence of the deviation of the radius of the grinding wheel, the movement of the grinding head and the grinding wheel, the deviation of the thickness of the part and the imbalance, it is necessary to isolate the corresponding terms in equation (8)

$$
\begin{aligned}
& d x=\frac{\left(h_{3} p+c_{3}\right) d L+\left(h_{3} p+c_{3}\right) d R-\left(h_{1} p+c_{1}\right) d s}{m_{1} p^{2}+h_{1} p+h_{3} p+c_{1}+c_{3}}+ \\
& +\frac{Q}{m_{1} p^{2}+h_{1} p+h_{3} p+c_{1}+c_{3}}
\end{aligned}
$$

which corresponds to the one shown in Fig. 2 functional diagram. 


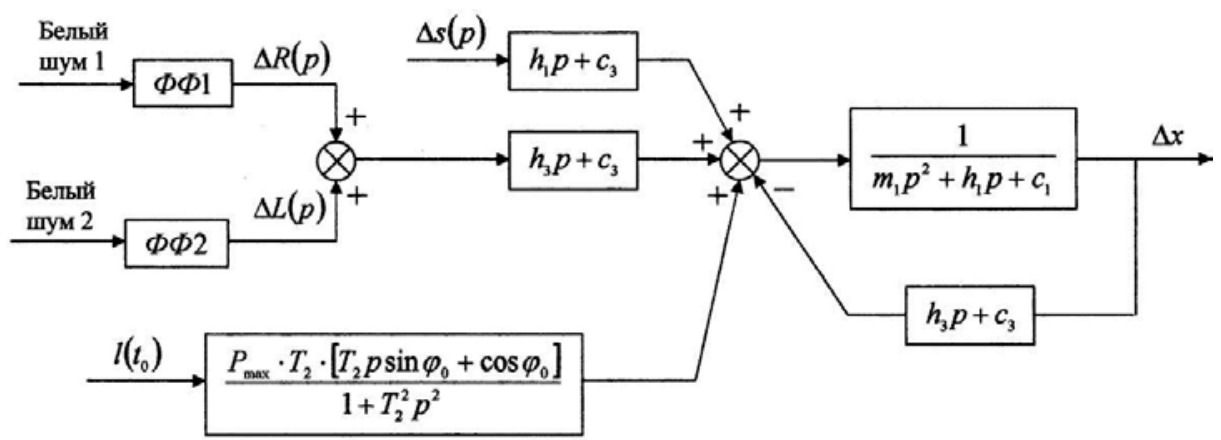

Fig. 2. Functional diagram of the dynamics of the process of flat external grinding.

Expression (9) allows us to present the following equity impacts of deviations from the design treatment regime in the deviation of the actual depth of cut $\Delta t_{f}$ :

- the influence of the deviation of the vertical feed of the grinding wheel $\Delta S$ from the nominal value $S_{0}$

$$
W_{d s}=\frac{-c_{1}-h_{1} p}{m_{1} p^{2}+\left(h_{1}+h_{3}\right) p+c_{1}+c_{3}}
$$

- the influence of the deviation of the shape of the grinding wheel $\Delta R$ from the nominal value $R_{0}$

$$
W_{d R}=\frac{h_{3} p+c_{3}}{m_{1} p^{2}+\left(h_{1}+h_{3}\right) p+c_{1}+c_{3}}
$$

- the influence of the deviation of the thickness of the workpiece $\Delta L$ from the nominal value $L_{0}$

$$
W_{d L}=\frac{h_{3} p+c_{3}}{m_{1} p^{2}+\left(h_{1}+h_{3}\right) p+c_{1}+c_{3}}
$$

- influence of imbalance

$$
W_{Q}=\frac{P_{\max } \cdot T_{2}}{1+T_{2}^{2} p^{2}} \cdot \frac{\left[T_{2} p \sin \varphi_{0}+\cos \varphi_{0}\right]}{m_{1} p^{2}+\left(h_{1}+h_{3}\right) p+c_{1}+c_{3}} .
$$

Relations (10) ... (13) can serve to develop a system of automatic stabilization of the depth of grinding.

When modeling the grinding process, it is necessary to obtain model data on the deviation of the radius of the grinding wheel from the nominal value. To solve such a problem it is necessary to develop a description of an equivalent dynamic link that converts a standard signal in the form of Gaussian white noise to a random process.

In order to take into account the influence of the statistical properties of the current deviations of the radius vectors of the grinding wheel and the thickness of the workpiece, in addition to the input signal, a random process with the same stochastic characteristics as for the current deviation of the radius vector of the grinding wheel and the workpiece 
topography. The corresponding random input signal can be obtained using a shaping filter $[3,4]$.

The principle of obtaining a random process with given parameters - the spectral characteristic (correlation function) is illustrated by the scheme (Fig. 3).

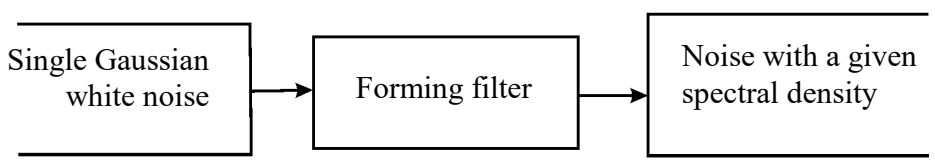

Fig. 3. Scheme of obtaining the noise with given parameters using a shaping filter.

The output coordinate of the shaping filter according to its statistical parameters corresponds to the parameters of the components of the dynamic system that characterizes the processes of the impact of the grinding wheel on the workpiece during grinding. For the settings of the forming filter, the results of statistical processing of profilograms of the working surface of the grinding wheel are required.

\section{Conclusion}

The developed mathematical model of the operation of flat external grinding can be used to determine the evaluation of the quality characteristics of machined parts and systems for automatic control of the process of flat external grinding when the model is supplemented by dynamic links that characterize the statistical properties of the grinding wheel and workpiece.

\section{References}

1. Lobanov D.V. Stress-strain state of hard-alloy cutting elements with diamond sharpening, Science Vector of Toliati state university. v. 3-1 (33-1), pp. 85-91 (2015).

2. Lobanov D.V. A technique for predicting the damage of a hard alloy when sharpening a tool for processing nonmetallic composites, Actual problems in mechanical engineering. v.1-2, pp. 78-84 (2018).

3. Soler Ia.I. Modeling of thermal physics of flat grinding. Problems of mechanical engineering and reliability of machinery, v. 5, pp. 56-62 (2005).

4. Bratan S.M. Automatic control of machining processes (Vuzovskiy uchebnik, Moscow, 2017).

5. Bratan S.M. Improving the quality of parts when grinding in floating workshops (Vuzovskiy uchebnik, Moscow, 2018).

6. Bratan S.M. Synthesis of the Kalman-Bussy filter for estimating the state of the grinding operation, Modern directions and prospects for the development of processing technology and equipment in engineering. FSFI HE «SevGU», pp. 87-91 (2015).

7. Vladeckaja E.A. Ensuring the quality of grinding by reducing external disturbances in a floating workshop. Fundamental and applied problems of technology and technology. v. 6 (314), pp.88-103 (2015).

8. Vladeckaya E.A. Development of a shaping filter that simulates the dynamics of sea waves in a floating repair shop, J. Sci. Instrument Making and Transport, v.150, pp. 36-40 (2014). 
9. Kharchenko A.O. Increase the accuracy of the round grinding process by efficient vibration isolation of the machine. International Scientific Conference on engineering design and research of automotive vehicles and machines «SAKON'08». - Rzeszow Przeclaw, Polska, 24-27 Wrzesein, v. 8. - pp. 47-58 (2008).

10. Kharchenko A.O. The reaction of the dynamic system to an arbitrary signal, using the example of a machine in the conditions of a floating workshop. Monografie «Maszyny i procesy produkcyjne» (Mashiny i proizvodstvennye processy).- Lublin, Polska: Politechnika Lubelska, pp. 86-98. (2015).

11. S. Bratan, A. Kolesov, S. Roshchupkin, T. Stadnik, Theoretical-probabilistic model of the rotary belt grinding process, MATEC Web of Conferences, V. 129, 01079 (2017).

12. Vladetskaya E.A. Modeling of vibration effects from external sources when grinding parts in laboratory conditions, Vestnik sovremennykh tekhnologiy: Sb. nauch. trudov.- Sevastopol': SevGU, V.4.- pp.25-34 (2016).

13. Yakimov A.V. Optimizatsiya protsessov shlifovaniya (Moscow, Mashinostroyeniye, 1975). 